\title{
Vigilância da hanseníase em Olinda, Brasil, utilizando técnicas de análise espacial
}

\author{
Leprosy surveillance in Olinda, Brazil, \\ using spatial analysis techniques
}

\author{
Tiago Lapa ${ }^{1}$ \\ Ricardo Ximenes ${ }^{2}$ \\ Nilza Nunes Silva 3 \\ Wayner Souza 1 \\ Maria de Fátima Militão Albuquerque 1 \\ Gisele Campozana ${ }^{1}$
}

\footnotetext{
1 Centro de Pesquisas Aggeu Magalhães, Fundação Oswaldo Cruz.

Av. Moraes Rego s/n, Cidade Universitária, C. P. 7472, Recife, PE 50670-420, Brasil. tiago@cpqam.fiocruz.br wayner@cpqam.fiocruz.br Militão@cpqam.fiocruz.br Giselle@cpqam.fiocruz.br 2 Departamento de Medicina Tropical, Centro de Ciências da Saúde. Av. Moraes Rego s/n, Cidade Universitária, Recife, $P E$ 50670-420, Brasil.

rximenes@elogica.com.br

3 Faculdade de Saúde Pública, Universidade de São Paulo. Av. Dr. Arnaldo 715, São Paulo, SP 01246-904, Brasil. nndsilva@usp.br
}

\begin{abstract}
In the State of Pernambuco, Brazil, leprosy has been mainly an urban disease, with an uneven geographical distribution related at least partially to the way urban space has been occupied and transformed. Spatial analysis may thus become an important tool to establish an epidemiological surveillance system for leprosy. Homogeneous micro-areas were defined in the city of Olinda through the integration of two databases, the Population Census and SINAN, and through the use of digital maps and geoprocessing techniques. Census tracts were classified according to a social deprivation index (SDI), and micro-area homogeneity was based on similar values for this indicator. Cluster analysis (K-means) was used to define cut-offs between strata. The same procedure was repeated using the income variable only. When the association was tested between the mean SDI value and the mean leprosy detection rate for the period 1991-1996, the value obtained for $r^{2}$ was $66.1 \%$ in the multiplicative model, increasing to $84.3 \%$ when the income variable was used. To define different intervention strategies, census tracts were regrouped in three levels of risk: high, moderate, and low. The methodology enabled the identification (within each health district) of groups and/or areas with different risk of leprosy, hence allowing for the definition of control measures.
\end{abstract}

Key words Spatial Analysis; Epidemiology Surveillance; Leprosy

Resumo Contribui-se com o sistema de Vigilância Epidemiológica em nível local por meio de análise espacial em razão do predomínio do caráter urbano da hanseníase em nosso meio e por sua distribuição não homogênea - em parte, pela forma de ocupação e transformação do espaço urbano. Trabalha-se o conceito de risco coletivo pela definição de micro-áreas homogêneas mediante o indicador de carência social, verificando-se sua coerência com padrões da distribuição da hanseníase obtidos da base de dados do SINAN para o Município de Olinda no período 199196. Para cada estrato, definido segundo níveis semelhantes dos indicadores, foi calculado o coeficiente de detecção médio para o período. Ao exame da associação entre carência social (risco) $e$ coeficiente de deteç̧ão de hanseníase obteve-se coeficiente de explicação de 66,1\% no modelo multiplicativo, acrescido para 84,3\% com a variável renda. Para atender à lógica de intervenção, definiram-se estratos de alto, médio e baixo risco nos distritos sanitários e área programática. A construção desses mapas mostrou-se útil a instrumentalizar o planejamento em nível local.

Palavras-chave Análise Espacial; Vigilância Epidemiológica; Hanseníase 


\section{Introdução}

O presente trabalho foi realizado com o objetivo de analisar a ocorrência de casos de hanseníase no Município de Olinda, durante o período de 1991 a 1996, segundo sua distribuição espacial, correlacionando-a às condições de vida da população, visando subsidiar novas estratégias de intervenção.

A hanseníase no Brasil é endemia de relevância para a Saúde Pública, apresentando alta prevalência, com mais de 100.000 casos de doentes em registro ativo, além de taxa de prevalência em torno de 6,4/10.000 habitantes. $O$ Brasil ocupa o primeiro lugar em número de casos na América Latina e é o segundo, entre os países de maior índice endêmico no mundo, só superado pela Índia. Tais números podem ter sido subestimados, se for considerado o subregistro decorrente tanto da qualidade da atenção quanto da acessibilidade aos serviços de saúde (MS, 1996; OPS, 1998).

O estado de Pernambuco, cuja taxa de detecção é de 3,56/10.000 habitantes, mostra grande concentração urbana na distribuição espacial da hanseníase, destacando-se a Região Metropolitana do Recife (RMR), que conta com $41,3 \%$ da população do Estado e responde por $81,5 \%$ dos casos registrados, correspondendo a alto coeficiente de detecção - em torno de 6,43/10.000 habitantes. Semelhante processo de concentração dos casos de hanseníase em áreas urbanas, assim como de outras doenças infecciosas e parasitárias, relaciona-se aos movimentos migratórios, ao caótico quadro socio-sanitário dos espaços urbanos e à estruturação da rede de serviços de saúde, que não dá resposta adequada a todo o espectro de problemas existentes (Albuquerque, 1987; Albuquerque et al., 1989).

No que se refere à organização de serviços de saúde - de acordo com os princípios e diretrizes que norteiam a proposta do Sistema Único de Saúde (SUS) -, o desafio que se impõe é a estruturação de novo modelo assistencial ágil, dinâmico e resolutivo, capaz de superar a dicotomia entre as ações voltadas à assistência à saúde individual e as medidas coletivas. Com relação ao controle de doenças e, no caso específico, da hanseníase necessita-se dispor de sistemas de informação que produzam conhecimento e gerem indicadores epidemiológicos e operacionais úteis para instrumentalizar a tomada de decisão nos diferentes níveis do sistema de saúde (Albuquerque, 1995; Scholten \& Lepper, 1991).

Em geral, os dados de morbidade obtidos através da notificação e os respectivos indica- dores epidemiológicos são avaliados para macro-áreas - Municípios/Estados -, representando a média de ocorrência dos eventos na região, o que dificulta a visualização de diferenciais de risco para menores níveis de agregação espacial - bairros, localidades, distritos - que representam micro-áreas de planejamento local (Daumerie, 1997).

Nos últimos anos, um levantamento bibliográfico em publicações nacionais - Revista de Saúde Pública e Cadernos de Saúde Públicaindica que 1/5 dos artigos na área de saúde pública, aproximadamente, fazem referência à questão da localização espacial, dos quais apenas $1 / 3$ avança da simples apresentação gráfica de apoio para algum tipo de comparação entre áreas pela associação de fatores com a ocorrência da doença.

Apesar de seu potencial, as técnicas de representação espacial são relativamente pouco utilizadas na saúde em decorrência, em parte, das dificuldades inerentes à manipulação deste tipo de informação, apesar da crescente disponibilização de tecnologias de tratamento das informações gráficas e de mapas em microcomputadores (Cruz, 1996).

No Brasil, o desenvolvimento dessas análises em saúde é dificultado pela restrita disponibilidade desses recursos na rede de serviços. O número de municípios que acumulou alguma experiência com esse tipo de abordagem é reduzido, mesmo que se examine a evolução em seu emprego, a julgar pelas apresentações em congressos e eventos diversos, inclusive aqueles da área de Sistema de Informações Geográficas - SIG - (GIS BRASIL, GISNORDESTE, entre outros).

Neste trabalho são explorados métodos de análise e de representação gráfica no espaço que venham a contribuir para a vigilância epidemiológica dos sistemas locais de saúde de modo a limitar a sua complexidade em função da viabilidade de uso, levando em conta as carências de estrutura e recursos a esse nível.

Um sistema que apresenta potencialidade de uso com abrangência universal é referido ao censo demográfico. Tal sistema contempla bases de dados que fornecem informações socioeconômicas das populações, além de outras, de caráter sanitário e ambiental, a respeito do conjunto dos setores censitários de todas as cidades brasileiras. O setor censitário é a célula mínima que serve de base à execução do censo e se compõe, na zona urbana, sempre que possível, de um conjunto de quarteirões com limites nítidos. O tamanho destes abarca, em média, 300 domicílios, abrangendo populações em torno de 1.500 habitantes, o que per- 
mite supor, na maioria das vezes, a existência de certa homogeneidade no tocante às características acima mencionadas (IBGE, 1993; Ximenes et al., 1999).

Assim, uma combinação possível de informações provenientes de diferentes sistemas teria lugar por meio de agregação da informação a respeito do setor censitário de residência de cada indivíduo a todo o sistema de informação que trate de eventos de saúde e, no caso da hanseníase, ao Sistema de Informação sobre Agravos de Notificação (SINAN). A partir daí estaria possibilitado o geo-referenciamento desses eventos e a análise espacial da distribuição de sua ocorrência, além da construção de indicadores de situações de risco para diferentes doenças e agravos. A construção desses indicadores será fortalecida mediante a integração de sistemas de informação que tomem em conta as características do espaço urbano e sejam fundamentados na lógica da homogeneidade/heterogeneidade socioeconômica-sanitária dos grupos populacionais neles residentes. O referenciamento a menores células - tal como o setor censitário - permitiria identificar territórios geográficos (grupos populacionais) que, por suas características, constituem prioridade para determinado programa ou atividade de vigilância, buscando-se maior impacto e racionalização de recursos (ABRASCO, 1997; Barcellos \& Santos, 1997; RIPSA, 1997; Ximenes et al., 1999).

Nesta perspectiva, o Grupo Técnico de Informação em Saúde Pública (GTISP), da Associação Brasileira de Pós-Graduação em Saúde Coletiva (ABRASCO), em 25 e 26/08/97, emitiu recomendações em seu relatório da oficina de trabalho - que fora organizada com vistas à compatibilização das bases de dados nacionais - entre as quais estão as de melhorar o registro das variáveis de localização do indivíduo, de eventos e de unidades de assistência, bem como a padronização dos dados de endereço dos diferentes sistemas. Sugeriu ainda o estudo de alternativas entre a Fundação Instituto Brasileiro de Geografia e Estatísticas (IBGE) e a Empresa Brasileira de Correios e Telégrafos (EBCT) para tornar compatíveis o Código de Endereçamento Postal (CEP) e o setor censitário, ademais da capacitação de recursos humanos (Barcellos \& Santos, 1997; RIPSA, 1997).

Este estudo adota o pressuposto de que a distribuição geográfica da hanseníase não é uniforme, havendo tendência à concentração dos casos em determinados espaços - regiões, cidades habitações etc. -, o que faria com que certas áreas estivessem sujeitas a risco diferenciado com relação ao adoecer (Daumerie, 1997; Fine, 1982, 1983). Além disso, e a exemplo de outras doenças infecciosas, a melhoria das condições de vida tem-se mostrado como forma eficiente de controle da hanseníase, estando relacionada, portanto, a sua distribuição, como bem atesta o seu desaparecimento do norte da Europa. Em que pese a dificuldade de avaliação dos mecanismos mediadores dessa relação, a sua associação com condições de habitação, estado nutricional e escolaridade é citada na literatura. Estes fatores, entre outros, poderiam potencializar a transmissão, por estarem ligados às condições de higiene ou por modularem a resposta do sistema imune (Van Beers et al., 1996).

\section{Material e métodos}

O estudo foi desenvolvido no Município de Olinda, Estado de Pernambuco, situado na Região Nordeste do Brasil. Olinda está localizada no litoral norte do estado, tem área aproximada de $40.83 \mathrm{~km}^{2}$, conta com 349.380 habitantes - de acordo com a contagem populacional do IBGE para 1996 (IBGE, 1998) - e apresenta densidade populacional de $8.553,59 \mathrm{hab} / \mathrm{km}^{2}$, uma das mais altas do país.

Os dados a respeito de casos novos de hanseníase detectados no período de 1991 a 1996 em residentes de Olinda foram obtidos do SINAN. O perfil socioeconômico-sanitário foi delineado a partir das informações do Censo Demográfico agregadas ao nível de setor censitário (IBGE, 1993).

Os setores censitários foram considerados unidade básica de análise por constituírem unidades espaciais com o maior nível de desagregação disponível e, portanto, relativamente mais homogêneas do que outros espaços geográficos, tais como bairros, distritos sanitários etc. (Rizzardi et al., 1993), para os quais há disponibilidade de dados censitários quanto aos fatores socioeconômicos e demográficos.

Foi utilizada a base territorial do município com o arruamento - confeccionada em 1988 -, obtida com a Fundação de Desenvolvimento Metropolitano (FIDEM), responsável pelo projeto UNIBASE, que tem por objetivo a construção de mapas digitais dos municípios da Região Metropolitana do Recife. A esta base foram adicionadas camadas relativas aos limites territoriais do Município, bem como aos Distritos Sanitários, Áreas Programáticas e Bairros, fundamentadas em mapas e informações colhidas junto ao IBGE, à Secretaria de Planejamento - Diretoria de Meio Ambiente de Olinda; Setor de Cartografia - e à Coordenadoria Regional de Pernambuco da Fundação Nacio- 
nal de Saúde (FUNASA). A partir desse novo mapa urbano de Olinda, digitalizou-se camada com os 243 setores censitários através do software MAXICAD (MAXIDATA, 1996), tomandose por base os descritores dos limites de setores censitários do IBGE - Censo/1991.

Para referenciar os endereços de cada caso de acordo com a base de dados do SINAN, trabalhou-se com o cadastro de logradouros da EBCT, com a Base de Dados do Reconhecimento Geográfico (RG) da Coordenação RegionalPernambuco da FUNASA - que consta de croquis com identificação de ruas, permitindo identificar novos trechos ou mesmo logradouros ainda não constantes das bases disponíveis -, com a Base de Dados de Levantamento Cadastral do Município de Olinda e com a Base de Dados com endereços da Companhia Telefônica de Pernambuco (TELPE) - que indica o número em que ocorrem os cruzamentos de ruas, de modo a identificar trechos.

Os casos de hanseníase e as informações socioeconômicas e demográficas foram geo-referenciadas através do software arcinfo/arcview.

O indicador composto "situação de risco coletivo" foi construído segundo metodologia proposta anteriormente (UNICEF, 1994), ao passo que a escolha das variáveis socioeconômicas do Censo-IBGE/91 resultou da revisão de literatura, selecionando-se aqueles trabalhos adequados à construção de indicadores de carência social e/ou relacionados à probabilidade de adoecer: renda, educação, abastecimento d'água, habitantes por dormitório, habitações em aglomerados subnormais e densidade demográfica.

Afora a técnica de utilização do indicador composto para a construção de estratos de risco a partir do conjunto de variáveis explicitadas anteriormente, foi trabalhada a opção de uso de variável única, a renda, para testar sua capacidade de explicação da ocorrência da doença. Esta simplificação do modelo facilitaria sua implementação em nível local, particularmente, no primeiro estágio de uso da técnica.

Considerando que o fator densidade populacional pode ter forte relação com a transmissão da hanseníase, assim como que a identificação dos setores de risco pode ter sofrido influência da renda, procedeu-se à revisão do mapa de risco em um segundo momento. O critério adotado foi o de retirar os setores do agrupamento de maior risco com base na renda quando evidenciam mais baixo risco com relação à densidade populacional, visando corrigir efeitos de diferenças geradas pela grande variação do fator extensão territorial e ocupação do espaço. Setores de maior risco, mas inte- grantes do último decil da distribuição da variável habitantes $/ \mathrm{km}^{2}$ foram retirados da condição de risco.

Com base nas estimativas populacionais para primeiro de julho dos anos em estudo, foram calculados os "coeficientes de detecção anual de casos” por 10.000 habitantes, observando-se sua magnitude e tendência e avaliando-os de acordo com o Instrumento de Avaliação do Programa Nacional de Eliminação da Hanseníase.

Com o objetivo de obter maior estabilidade dos dados, trabalhou-se com o coeficiente de detecção médio para o período, obtido pelo quociente entre a média anual de casos novos e a população estimada por interpolação geométrica para o meio do período $31 / 12 / 93$.

\section{Análise dos dados}

Para a detecção da existência de cluster espacial, utilizou-se o teste de Moran's I e IPOP, método que usa coeficiente de correlação dos pesos de conectividade entre os setores estudados.

Dispondo-se dos escores médios - $\mathrm{Z}$ - indicador de carência social -, bem como dos escores para a variável indicadora da renda (variáveis independentes) para cada setor censitário, estes foram agregados de acordo com os decis de suas distribuições, de modo a fornecer maior estabilidade, procedendo-se à análise de correlação com o coeficiente de detecção (variável dependente).

Em etapa subseqüente, a técnica de K-médias, foi empregada para obtenção de três agrupamentos, classificados de alto, médio e baixo risco, obtendo-se estratos que atendam à lógica da intervenção (Marshal, 1991).

Considerando os efeitos decorrentes das variações entre as dimensões territoriais dos setores censitários e o seu grau de ocupação (urbanização), foram identificados aqueles de grandes áreas, mas com baixa "densidade populacional”. Reavaliou-se então o risco, ponderando-se a influência dessa variável não disponível no Censo Demográfico. A densidade populacional, medida pela razão de habitantes por área do setor (hab/ $\mathrm{km}^{2}$ ), pôde ser estimada após a digitalização dos polígonos representativos dos setores censitários, quando, através do software de geoprocessamento, foi possível conhecer a área de cada setor. 


\section{Resultados}

O município apresenta altos e crescentes "coeficientes de detecção anual de casos” ao longo do período estudado, como mostra a Figura 1.

A Figura 2 mostra as camadas de setores censitários do Município de Olinda e dos limites dos Distritos Sanitários e Áreas Programáticas, podendo ser vistos ainda os bairros, a localização das unidades de saúde e as principais vias de acesso.

Tendo em vista as deficiências do sistema, a informação concernente ao endereço era inadequada ou incompleta para alguns casos, acarretando a identificação precisa do setor censitário de $85,5 \%$ dos casos. Nos 14,5\% restantes, embora não tenha sido possível localizar com precisão o setor de residência, os dados disponíveis para a maioria deles forneciam evidências de que se tratava de residências edificadas em áreas de maior deficiência de estrutura urbana, tais como assentamentos recentes e favelas.

Na aplicação do teste para a verificação de existência de cluster obteve-se um Morans' I = 0,255879 , um Z-score $=8,695180$, resultado altamente significante $(\mathrm{p}<0,0001)$, indicando a existência de agrupamentos espaciais, também sugerido pelo IPOP $=0,0018753$, IPOP' = 0,4650065 e Z-score $=74,0551601$.

As Tabelas 1 e 2 expõem os decis da distribuição do indicador de carência social e renda e os valores dos coeficientes de detecção médio de hanseníase.

Os resultados do estudo de análise de variância para a verificação da associação entre o risco coletivo e a detecção da hanseníase segundo os decis da distribuição do Indicador de Carência Social (ICS) apresentaram coeficiente de explicação da ordem de $66,07 \%$ no modelo multiplicativo, de $29,71 \%$ no linear e de $34,99 \%$ no exponencial.

Estes resultados indicam que o modelo multiplicativo ajusta-se melhor à distribuição, sugerindo que o indicador de carência social pode explicar, até certo ponto, a ocorrência da hanseníase no Município de Olinda durante o período estudado. Considerando-se a segunda opção adotada, foram obtidos coeficientes de explicação da ordem de $84,32 \%$ para o modelo multiplicativo, $58,78 \%$ para o modelo exponencial e $56,90 \%$ para o linear ao usar a variável renda, indicando também que o modelo multiplicativo seria o mais adequado ao ajustamento da distribuição.

Na comparação entre a detecção média tanto com o ICS como com a renda, observaramse coeficientes de detecção menores que o es-
Figura 1

Coeficiente de detecção anual de hanseníase. Olinda, 1991-1996.

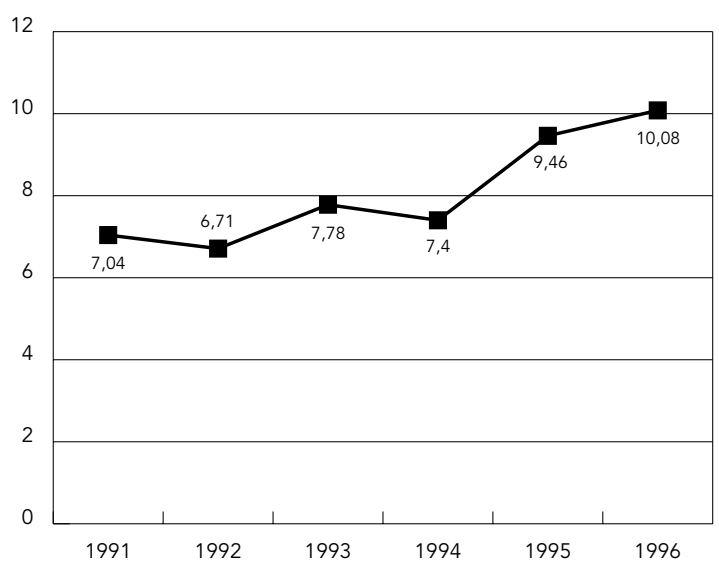

Figura 2

Mapa do Município de Olinda com os limites dos setores censitários, bairros, áreas programáticas e distritos sanitários - 1996.

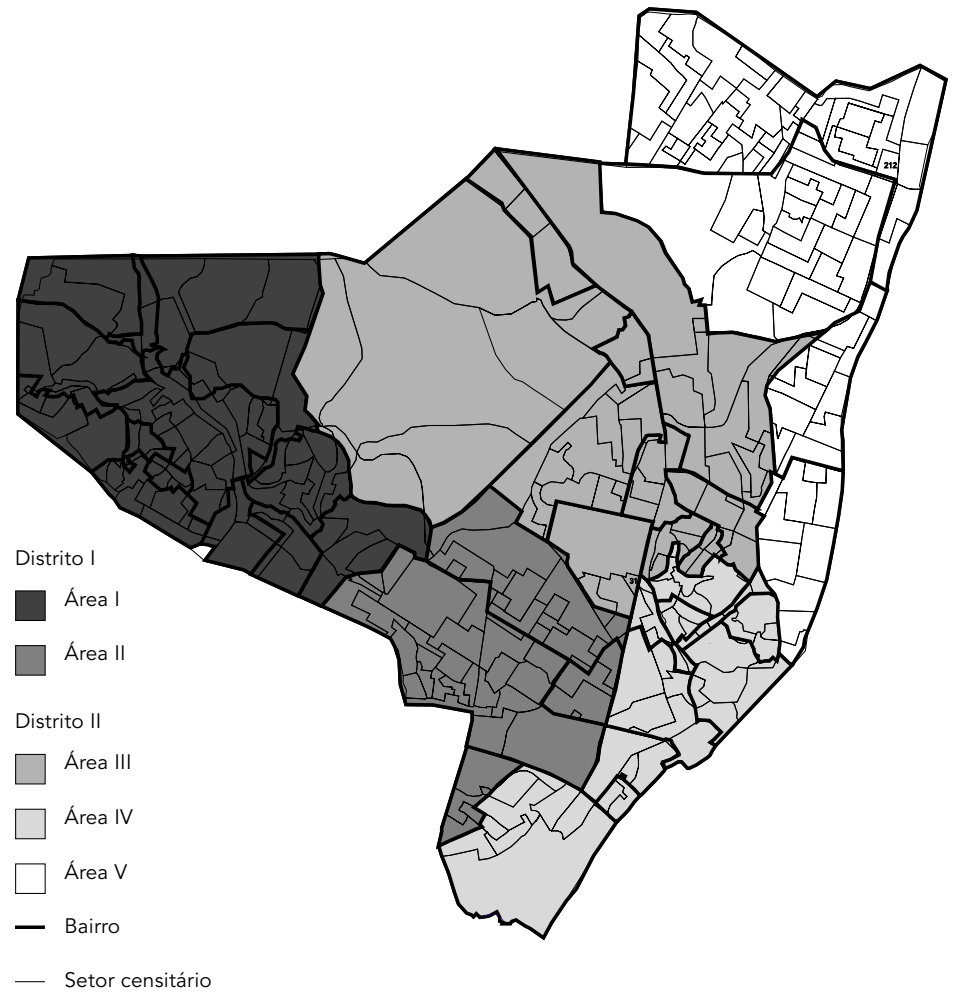


Tabela 1

Distribuição do coeficiente de detecção médio da hanseníase e indicador de carência social - Z por decil da distribuição do ICS. Olinda, 1991-1996.

\begin{tabular}{rrrrrr}
\hline Decil & Casos & $\begin{array}{l}\text { Média } \\
\text { de casos }\end{array}$ & $\begin{array}{l}\text { População } \\
(31 / 12 / 93)\end{array}$ & $\begin{array}{l}\text { Coeficiente } \\
\text { de detecção } \\
\text { média }\end{array}$ & Z \\
\hline 1 & 42 & 7,0 & 29.335 & 2,4 & 4,9 \\
2 & 79 & 13,2 & 33.598 & 3,9 & 9,3 \\
3 & 103 & 17,2 & 31.511 & 5,4 & 12,3 \\
4 & 113 & 18,8 & 33.473 & 5,6 & 15,5 \\
5 & 175 & 29,2 & 32.652 & 8,9 & 20,6 \\
6 & 218 & 36,3 & 35.452 & 10,2 & 25,4 \\
7 & 214 & 35,7 & 39.186 & 9,1 & 31,1 \\
8 & 153 & 25,5 & 36.629 & 7,0 & 38,8 \\
9 & 181 & 30,2 & 38.034 & 7,0 & 48,1 \\
10 & 154 & 25,7 & 35.281 & 7,3 & 58,4 \\
\hline
\end{tabular}

ICS = indicador de carência social.

Tabela 2

Distribuição do coeficiente de detecção médio da hanseníase e indicador de renda por decil da distribuição da renda. Olinda, 1991-1996.

\begin{tabular}{rrrrrr}
\hline Decil & Casos & $\begin{array}{l}\text { Média } \\
\text { de casos }\end{array}$ & $\begin{array}{l}\text { População } \\
(31 / 12 / 93)\end{array}$ & $\begin{array}{l}\text { Coeficiente } \\
\text { de detecção } \\
\text { média }\end{array}$ & Renda \\
\hline 1 & 32 & 5,3 & 28.568 & 1,9 & 0,05 \\
2 & 87 & 14,5 & 33.257 & 4,4 & 0,14 \\
3 & 96 & 16,0 & 34.313 & 4,7 & 0,21 \\
4 & 95 & 17,7 & 33.913 & 4,7 & 0,26 \\
5 & 160 & 26,7 & 32.628 & 8,2 & 0,33 \\
6 & 219 & 36,5 & 33.988 & 10,7 & 0,41 \\
7 & 157 & 26,2 & 37.851 & 6,9 & 0,50 \\
8 & 199 & 33,2 & 36.642 & 9,1 & 0,57 \\
9 & 224 & 33,7 & 38.725 & 9,6 & 0,67 \\
10 & 163 & 27,2 & 35.262 & 7,7 & 0,79 \\
\hline
\end{tabular}

perado nos últimos decis, correspondentes à população de mais baixa condição sócio-econômica, cuja estrutura urbana é mais precária.

Os mapas que indicam setores de alto, médio e baixo risco - agrupados através de técnica de K-médias de acordo com o indicador de carência social-Z e com a renda - estão apresentados na Figura 3, em que se observa concordância das duas classificações para $96,7 \%$ do total de setores estudados. Quanto aos coeficientes de detecção, os valores obtidos respectivamente para os estratos de alto, médio e baixo risco foram de $7.57,7.13$ e 1.92 por 10.000 habitantes, situação em que os agrupamentos foram baseados no indicador de carência social, e de 8.8, 8.2 e 3.9, quando os estratos foram definidos pela renda.

Na Figura 4 são apresentadas as áreas de risco no interior de cada Área Programática, distribuídas em setores de alto e médio risco com fundamento no contigente populacional, permitindo a definição de prioridades em consonância com a lógica de organização dos serviços.

A revisão do mapa de risco relativo à renda fundamentado na inserção da variável densidade populacional dos setores censitários (hab $/ \mathrm{km}^{2}$ ) produziu novo mapa do risco e redefinição das áreas prioritárias. Nessa nova classificação, como pode ser visto na Figura 5, observam-se importantes inversões na classificação anterior, como a da Área II que sai da prioridade três para a prioridade um em razão de sua menor área, embora conte com grande densidade populacional, além de tratar-se, conforme depoimentos de técnicos da Secretaria Municipal, de área de acentuada deficiência de estrutura urbana e de baixa renda. A situação se inverte na Área III, caindo a prioridade de 2 para 4. Os coeficientes de detecção calculados por área programática são os apresentados a seguir: Área I = 54,1; Área II = 77,9; Área III = 23,9; Área IV $=45,2$; Área V = 17,9.

\section{Discussão}

Os resultados do estudo indicam que a distribuição espacial heterogênea da hanseníase em Olinda não é aleatória, identificando-se um padrão de agregação no espaço que está associado às condições de vida da população e é expresso mediante o indicador de carência social - calculado a partir de escores relativos a variáveis socioeconômicas do censo demográfico ou expresso apenas através da variável renda.

$\mathrm{Na}$ análise dos dados, as dificuldades encontradas na identificação do setor censitário de residência - unidade espacial usada no estudo -, que impede o geo-referenciamento de $15 \%$ dos casos, podem ser a explicação para o achado de baixos coeficientes de detecção da hanseníase em alguns estratos de maior carência social. Indubitavelmente, pelas características dos endereços fornecidos, esses casos seriam alocados nos decis correspondentes às condições sócio-econômicas mais precárias. Isso se refletiria no cálculo do coeficiente de explicação, sobretudo com relação ao modelo linear que, com essa exclusão, estaria subestimado. 

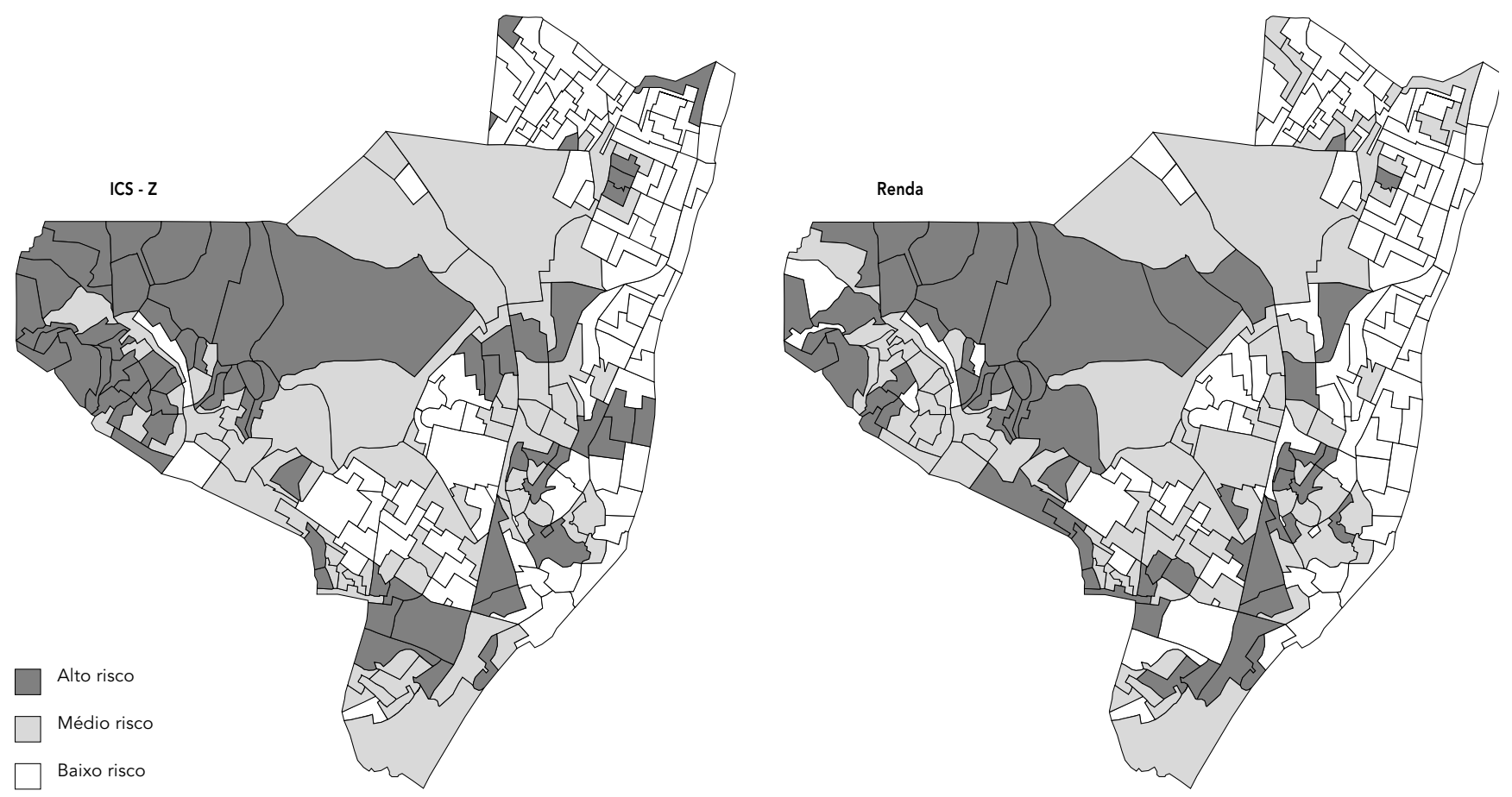

\begin{tabular}{lcc}
\hline Estrato & \multicolumn{2}{c}{ Número de setores censitários } \\
& Estratificação pelo ICS & Estratificação pela renda \\
\hline Alto risco & 61 & 60 \\
Médio risco & 77 & 85 \\
Baixo risco & 103 & 96 \\
\hline
\end{tabular}

ICS = indicador de carência social.

Outro aspecto a ser levado em conta é o da acessibilidade aos serviços, cuja dificuldade atinge, via de regra, os mais carentes. Ressaltese que $45,02 \%$ da população de Olinda possui renda insuficiente, ou seja, essa proporção de indivíduos conta com renda familiar per capita inferior a 50\% do salário mínimo de 1/7/91 (PNUD, 1998), o que situa a luta pela sobrevivência como necessidade fundamental e faz com que outras necessidades básicas sejam relegadas a segundo plano. A esse quadro sobrepõe-se a precariedade do serviço de transportes urbanos. A forma de inserção dos adultos no mercado formal ou informal de trabalho impede seu acesso aos serviços de saúde ou os exclui.

$\mathrm{O}$ instrumento empregado para estratificar o município em áreas de risco é útil na defini- ção de prioridades das ações de saúde, apoiando o planejamento de diferentes estratégias adequadas a situações específicas e permitindo maior racionalização na alocação de recursos (OPAS, 1996).

Outro obstáculo enfrentado para o geo-referenciamento diz respeito à desatualização das bases digitais, considerando-se que a base digital de Olinda usada no presente estudo foi gerada por aerofotogrametria e data de 1988, bem como que a base cadastral disponível não apresenta atualização de logradouros, ocasionando dificuldades na identificação do setor censitário relativo a alguns endereços registrados no SINAN (Guerra \& Carvalho, 1998).

Apesar de os dados sócio-econômicos e demográficos do Censo/IBGE serem coletados 
Figura 4

Mapa com os estratos de risco com base na variável renda, identificando prioridades por área programática a partir do contingente populacional de residentes em setores de alto e médio risco. Olinda, Pernambuco, Brasil.

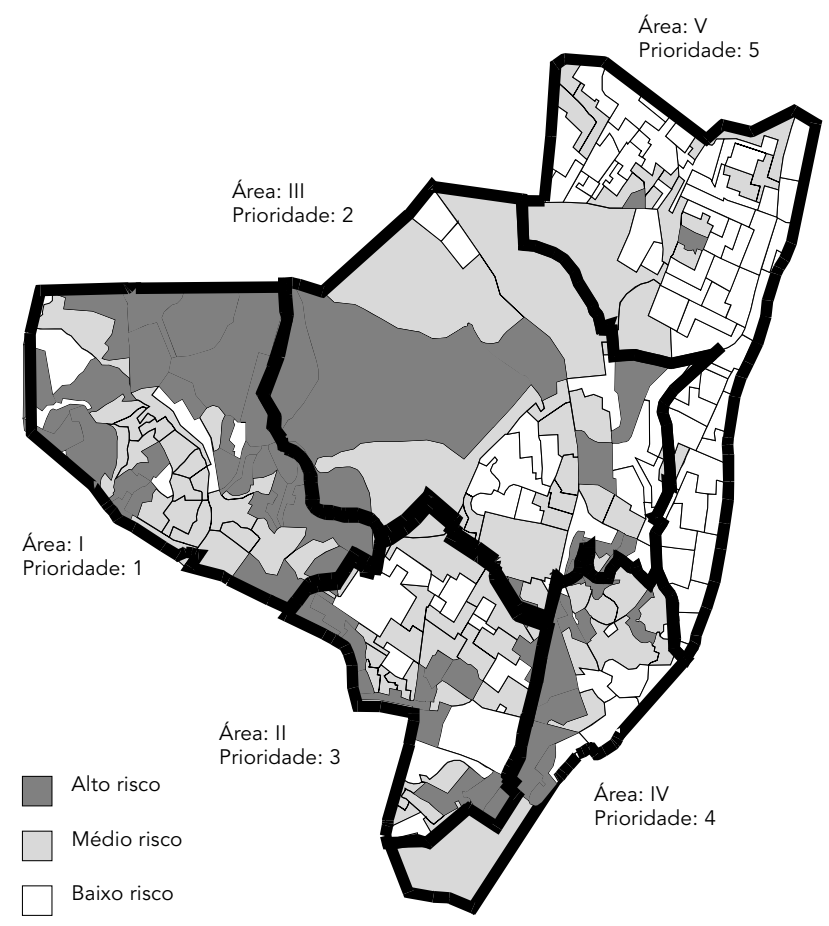

\begin{tabular}{lccc}
\hline $\begin{array}{l}\text { Área } \\
\text { programática }\end{array}$ & $\begin{array}{l}\text { População } \\
\mathbf{3 1 / 1 2 / 9 3}\end{array}$ & $\begin{array}{l}\text { População em } \\
\text { setores de alto } \\
\text { e médio risco }\end{array}$ & $\begin{array}{l}\text { \% de População } \\
\text { em setores de alto } \\
\text { e médio risco }\end{array}$ \\
\hline I & 83.661 & 58.993 & 70,5 \\
II & 65.085 & 50.846 & 78,1 \\
III & 70.400 & 55.670 & 79,1 \\
IV & 28.771 & 25.396 & 88,3 \\
V & 97.234 & 24.828 & 25,5 \\
Total & 345.151 & 215.733 & 62,5
\end{tabular}

em periodicidade que não oferece perfeita correspondência com os dados de morbidade, acredita-se não ter ocorrido, durante o período estudado, mudanças consideráveis no perfil socioeconômico demográfico que pudesse prejudicar de modo substancial os resultados do estudo (Van Oers \& Reelikm, 1992). Neste aspecto, cabe comentar que Olinda preserva relativa estabilidade em termos de ocupação do espaço, diferentemente de outras cidades. Sua conformação básica, urbana não apresenta alterações significativas, mantendo a antiga ca- racterística de cidade dormitório, dependente da capital.

A construção do ICS de acordo com a metodologia adotada não introduz ponderações relativas à influência de cada uma das variáveis associadas à carência (D'Orsi \& Carvalho, 1998). Esta consideração poderia ser apontada como limitação, mas é justamente em virtude de sua simplicidade e factibilidade para a operacionalização em nível local que, aliada à adequação observada neste estudo, legitima sua utilização.

A opção de adotar diferentes estratégias, avaliando-se a capacidade do ICS e da renda no tocante a discriminar estratos de risco associados à ocorrência da doença, decorreu da intenção de testar diferentes metodologias, visando à adequação de uso no nível dos sistemas locais de saúde. O emprego de diferentes técnicas aplicadas à situação de tuberculose, descrito em outro artigo (Souza, 1998), complementa esta estratégia.

Um fato relevante a ser observado neste contexto consistiu nos resultados obtidos a partir da análise de concordância do coeficiente de detecção médio da hanseníase com o indicador de carência social e com a variável renda, validando, de certa forma, o modelo proposto.

O uso do setor censitário como unidade básica de estudo - considerando-se sua condição de menor unidade para a qual se dispõe de dados sócio-econômicos, configurando estudos em pequenas áreas - gerou um desafio imposto pela questão da estabilidade dos dados e, como forma de contornar o problema, adotouse a agregação temporal e espacial. A opção mostrou-se adequada ao considerar a heterogeneidade de unidades maiores - como, por exemplo, o bairro -, o que possibilitou um reagrupamento em estratos mais homogêneos, não se respeitando os limites das tradicionais divisões, as quais nem sempre atendem ou respeitam os critérios de interesse.

No que diz respeito ao estabelecimento dos pontos de corte para a composição dos estratos de risco, trabalhou-se com três estratos na perspectiva da definição de prioridades para orientar as estratégias de intervenção, compondo-se grupos relativamente homogêneos que apresentaram coerência com os coeficientes de detecção. Considerou-se uma proposta de intervenção na lógica do serviço, com enfoque em modelo assistencial para o nível local, tendo em conta as dificuldades em operacionalizar mais de três estratégias em áreas programáticas. Esta opção atende inclusive a expectativa dos profissionais atuantes em Olin- 
da, adequando-se ao confronto com quadro subjetivo.

No uso dos mapas de padrões, o efeito visual pode induzir à má interpretação, levando a conclusões errôneas acerca dos resultados apresentados. Assim, foi realizada uma ponderação com a extensão territorial, atentando-se para a ocupação do espaço correspondente aos setores censitários, o que resultou em mapa equalizado do risco (Carvalho, 1997).

\section{Conclusões}

Os indicadores construídos segundo a lógica de situação de risco coletivo, pelos quais seja possível definir áreas ou estratos populacionais mais homogêneos quanto às características que determinam maior probabilidade de adoecer, ajudam não só a compreensão do processo de produção das doenças em função da ocupação do espaço urbano, como podem dar suporte à concepção de um sistema integrado de vigilância à saúde.

O uso dos SIG na saúde possibilita associar informações de diversas bases de dados em razão de sua capacidade integradora, contribuindo com o avanço das análises espaciais para os sistemas de vigilância à saúde. A produção de "mapas" que permitem facilmente visualizar situações de risco à saúde resultantes da interseção e da complementaridade de eventos, é coerente com um conceito epidemiológico de vigilância do espaço.

A utilização de bases de dados ambientais e sanitárias que integram dados do SINAN e do Censo/IBGE, como o que foi realizado no presente trabalho, indica a possibilidade de interações importantes, ressaltando-se o papel das ações intersetoriais na melhoria da qualidade da captação, registro e disponibilização das informações que são úteis ao planejamento e ao controle da ações de saúde.

A qualificação de áreas com base em indicadores de risco coletivo permite enxergar o município em partes heterogêneas, nas quais as ações podem ser planejadas em harmonia com critérios de necessidades e prioridades em nível local, guardando coerência com as diretrizes do SUS.

Sugere-se a utilização, no caso de Olinda, da forma simplificada do modelo. A partir daí, um processo gradual da intensificação de seu uso poderá conduzir à elaboração de modelos mais complexos, consolidando a incorporação de tecnologia ao avanço da qualidade dos sistemas de saúde.

\section{Figura 5}

Mapa com os estratos de risco com base na variáveis renda e hab/ $\mathrm{km}^{2}$, identificando prioridades por área programática a partir do contingente populacional de residentes em setores de alto e médio risco. Olinda, Pernambuco, Brasil.

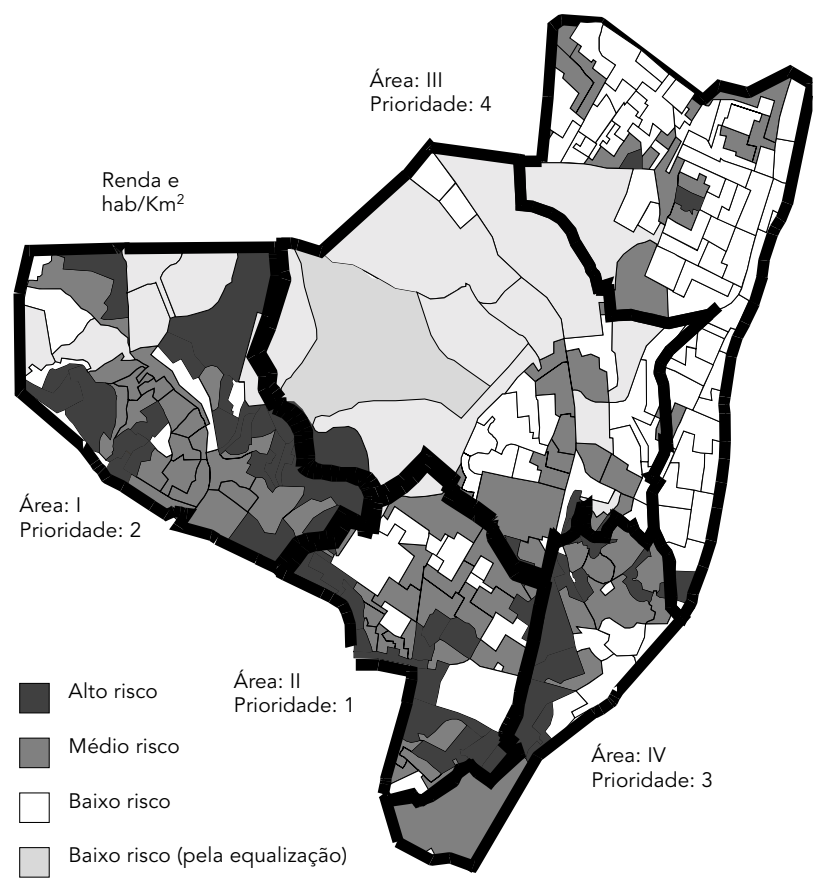

\begin{tabular}{lccc}
\hline $\begin{array}{l}\text { Área } \\
\text { programática }\end{array}$ & $\begin{array}{l}\text { População } \\
\mathbf{3 1 / 1 2 / 9 3}\end{array}$ & $\begin{array}{l}\text { População em } \\
\text { setores de alto } \\
\text { e médio risco }\end{array}$ & $\begin{array}{l}\text { \% de População } \\
\text { em setores de alto } \\
\text { e médio risco }\end{array}$ \\
\hline I & 83.661 & 37.596 & 44,9 \\
II & 65.085 & 42.430 & 65,2 \\
III & 70.400 & 23.000 & 32,7 \\
IV & 28.771 & 25.164 & 87,5 \\
V & 97.234 & 22.758 & 23,4 \\
Total & 345.151 & 150.948 & 43,7 \\
\hline
\end{tabular}




\section{Referências}

ABRASCO (Associação Brasileira de Pós-Graduação em Saúde Coletiva), 1997. Compatibilização de bases de dados nacionais. Informe Epidemiológico do SUS, 6:25-33.

ALBUQUERQUE, M. F. P. M., 1987. A Hanseníase no Recife: Um Estudo Epidemiológico para o Período 1960-1985. Dissertação de Mestrado, Recife: Faculdade de Medicina, Universidade Federal de Pernambuco.

ALBUQUERQUE, M. F. P. M., 1995. Urbanização, Favelas e Endemias: A Produção e o Controle da Filariose Bancroftiana no Recife. Tese de Doutorado, Rio de Janeiro: Escola Nacional de Saúde Pública, Fundação Oswaldo Cruz.

ALBUQUERQUE, M. F. P. M.; MORAIS, H. M. M. \& XIMENES, R., 1989. A expansão da hanseníase no Nordeste brasileiro. Revista de Saúde Pública, 23: 107-116.

BARCELLOS, C. \& SANTOS, S. M., 1997. Colocando dados no mapa: A escolha da unidade espacial de agregação e integração de bases de dados em saúde e ambiente através do geoprocessamento. Informe Epidemiológico do SUS, 6:21-29.

CARVALHO, M. S., 1997. Aplicação de Métodos de Análise Espacial na Caracterização de Áreas de Risco à Saúde. Tese de Doutorado, Rio de Janeiro: Instituto Alberto Luiz Coimbra de Pós-graduação e Pesquisa de Engenharia, Universidade Federal do Rio de Janeiro.

CRUZ, O. C., 1996. Homicídios no Estado do Rio de Janeiro: Análise da Distribuição Espacial e sua Evolução. São Paulo, 1996. Dissertação de Mestrado, São Paulo: Faculdade de Saúde Pública, Universidade de São Paulo.

D'ORSI, E. \& CARVALHO M. S., 1998. Perfil de nascimentos no município do Rio de Janeiro: Uma análise espacial. Cadernos de Saúde Pública, 14 (Sup.2):367-379.

DAUMERIE, D., 1997. Monitoring the Elimination of Leprosy. Foz do Iguaçu: Brazilian Association of Hansenology. (mimeo.)

FINE, P. E. M., 1982. Leprosy: The epidemiology of a slow bacterium. Epidemiologic Reviews, 4:161-168.

FINE, P. E. M., 1983. Natural history of leprosy - Aspects relevant to a leprosy vaccine. International Journal of Leprosy, 51:553-555.

GUERRA, H. \& CARVALHO, M. S., 1998. Geoprocessamento em epidemiologia. In: Congresso Brasileiro de Epidemiologia, Relatório da Oficina de Trabalho. Rio de Janeiro: ABRASCO.

IBGE (Fundação Instituto Brasileiro de Geografia e Estatística), 1993. Censos Demográficos 1991. Resultados do Universo Relativo às Características da População e dos Domicílios. Rio de Janeiro: IBGE.

IBGE (Fundação Instituto Brasileiro de Geografia e Estatística), 1998. Censos Demográficos. 15 de julho de 1998 <http://www.ibge.gov/censos>.

MARSHALL, R. J., 1991. A review of methods for the statistical analysis of spatial patterns of disease. Journal of the Royal Statistical Society, 154:221241.

MAXIDATA, 1996. Sistemas para Geoprocessamento. MAXICAD e DBMAPA for Windows. Curitiba: MAXIDATA.
MS (Ministério da Saúde), 1996. Instrumento de Avaliação do Programa Nacional de Controle e Eliminação da Hanseníase. Brasília: Coordenação Nacional de Dermatologia Sanitária, Fundação Nacional de Saúde.

OPAS (Organização Pan-Americana da Saúde), 1996. Hanseníase Hoje. Brasília: OPAS.

OPS (Organización Panamericana de la Salud), 1998. Hanseníase. 11 agosto $1998<\mathrm{http}$ ://www.opas. org.br/programas/hanseníase/hansen.htm>.

PNUD (Programa das Nações Unidas para o Desenvolvimento), 1998. Atlas de Desenvolvimento $\mathrm{Hu}$ mano. CD-ROM. Belo Horizonte: PNUD/Instituto de Pesquisas Econômicas Aplicadas/Fundação João Pinheiro/Fundação Instituto Brasileiro de Geografia e Estatística.

RIPSA (Rede Integrada de Informações Para a Saúde), 1997. Compatibilização de sistemas e base de dados (CBD) da Rede Integrada de Informações para a saúde (RIPSA) - Informe final, 1997. Informe Epidemiológico do SUS, 6:35-41.

RIZZARDI, M.; MOHR, M. R.; MERRILL, D. W. \& SELVIN, S., 1993. Interfacing U. S. census map files with statistical graphics software: Application and use in epidemiology. Statistics in Medicine, 12:1953-1964.

SCHOLTEN, H. J. \& LEPPER, M. J. C., 1991. The benefits of the application of geographical information systems in public and environmental health. Rapport Trimestrel de Statistique Sanitaire Mondiale, 44:160-170.

SOUZA, W. V., 1998. O Uso de Informações Sócio-econômicas na Construção de Indicadores de Situação Coletiva de Risco para a Ocorrência da Tuberculose em Olinda, Estado de Pernambuco. Dissertação de Mestrado, Recife: Núcleo de Estudos em Saúde Coletiva, Fundação Oswaldo Cruz.

UNICEF (Fundo das Nações Unidas para a Infância), 1994. Municípios Brasileiros: Crianças e suas Condições de Sobrevivência. Brasília: Fundação Instituto Brasileiro de Geografia e Estatística.

VAN BEERS, S. M.; DE WIT, M. Y. L. \& KLASTER, P. R., 1996. The epidemiology of Mycobacterium leprae: Recent insight. FEMS Microbiology Letters, 136: 221-230.

VAN OERS, J. M. \& REELICKM, N. F., 1992. Quantitative indicators for a healthy city-the Rotherdam local health information system. Journal of Epidemiology and Community Health, 46:293-296.

XIMENES, R. A. A.; MARTELLI, C. M. T.; SOUZA, W. V.; LAPA, T. M.; ALBUQUERQUE, M. F. M.; ANDRADE, A. L. S. S.; MORAIS NETO, O. L.; ALMEIDA-E SILVA, S. \& PORTUGAL, J. L., 1999. Vigilância de doenças endêmicas em áreas urbanas: A interface entre mapas de setores censitários e indicadores de morbidade. Cadernos de Saúde Pública, 15:53-61. 